\title{
PERITONITIS AGUDA TRAS CISTECTOMÍA RADICAL. EXPERIENCIA EN NUESTRO HOSPITAL Y REVISIÓN DE LA BIBLIOGRAFÍA.
}

Joaquín Navarro Gil, José Manuel Sánchez Zalabardo, José Carlos Salinas Payer', Raquel Bustamante Rodríguez² y José Gabriel Valdivia Uría.

Servicio de Urología, Servicio de Cirugía General' y Unidad de Cuidados Intensivos². Hospital Clínico Universitario "Lozano Blesa". Zaragoza. España.

\begin{abstract}
Resumen.- OBJETIVO: Las características de los pacientes que actualmente se intervienen podrían favorecer la aparición de complicaciones (tumores avanzados, pluripatología, etc.). Teniendo en cuenta la elevada morbimortalidad del proceso, creemos necesario conocer las características principales de la peritonitis aguda postoperatoria en nuestro área quirúrgica.
\end{abstract}

MÉTODOS: Se estudian las cistectomías radicales realizadas que presentaron en el postoperatorio peritonitis. Las variables a estudio fueron clasificadas en cuatro categorías: fisiología del paciente, intervención, peritonitis y morbimortalidad.

RESULTADOS: En el plazo estudiado, 9 de las 80 cistectomías se complicaron con peritonitis (1 1,25\%). Todos ellos varones por encima de 50 años excepto un paciente de 44 años. La intención quirúrgica fue en 5 de ellos curativa y paliativa en 4.5 de las peritonitis aparecieron dentro de la primera semana. El mecanismo inicial fue por dehiscencia, necrosis o hiperpresión 16,2 y 1 respectivamente) y el origen fue en la anastomosis urétero-ileal en 6 pacientes, en 2 la anastomosis intestinal y en 7 la ureterostomía cutánea. 3 de los 9 pacientes fallecen consecuencia de la peritonitis $13,75 \%$ del totall

CONCLUSIONES: La incidencia de las complicaciones intraabdominales en el postoperatorio inmediato de las cistectomías radicales es elevada y muchas veces difícil de diagnosticar. En los pacientes sometidos a esta intervención con carácter paliativo, la posibilidad de presentar complicaciones es mayor. La actuación precoz en estos casos es fundamental para corregir el cuadro clínico, condicionando el pronóstico del paciente.

Palabras clave: Cistectomía. Peritonitis. Complicaciones postoperatorias.

Summary.- OBJECTIVES: The characteristics of the patients may favour the appearance of complications. Taking into account the high morbimortality of the process, we believe that is convenient knowing main features of acute peritonitis in postoperatory in our surgical area.

METHODS: We have retrospectively studied the radical cystectomies having postoperative peritonitis. Variables considered can be classified in four main categories: patient physiology, operation, peritonitis and morbimortality.

RESULTS: During considered time, 9 of 80 cystectomies showed complications related with peritonitis (1 1,25\%). Except one patient, 44-year-old, all others where 50- 
year-old or older males. 5 surgeries were curative and 4 palliative. 5 of the peritonitis appeared during first week. Initial mechanism was dehiscence, necrosis or hypertension (6.2 and 1 respectivelly). In 6 patients, origin was ureteroileal anastomosis, 2 intestinal anastomosis and 1 cutaneous ureterostomy. 3 of 9 patients died due to peritonitis.

CONCLUSIONS: The incidence of intra-abdominal complications during the immediate post operative period of radical cystectomy is high and, often, difficult to diagnose. In patients subjected to this surgery as a palliative method, possibility of showing complications is higher. To correct this medical profile, early intervention is fundamental, determining patient prognosis.

Keywords: Cystectomy. Peritonitis. Postoperatory complication.

\section{INTRODUCCIÓN}

La peritonitis postoperatoria es una de las complicaciones más graves y frecuentes que se presenta tras la cirugía abdominal. Se trata de una entidad con alta morbilidad provocando un importante retroceso en la evolución quirúrgica que, en ocasiones, deriva en la muerte del paciente. De hecho, la mortalidad de la peritonitis postoperatoria viene estimada entre el $22 \%$ y el $55 \%$, mayor que la secundaria a una perforación espontánea de una víscera intraabdominal (entre el $11 \%$ y el $29 \%$ ).

Entre las diferentes intervenciones que se desarrollan en la cavidad peritoneal (digestivas, urológicas, vasculares...), la cirugía del aparato digestivo es la que más frecuentemente se complica con peritonitis postoperatoria, siendo una complicación excepcional en pacientes intervenidos por patología urológica. No es sencillo encontrar en la literatura médica artículos que se centren en las infecciones intraabdominales tras el tratamiento quirúrgico de causa urológica.

Teniendo en cuenta la elevada morbimortalidad del proceso, creemos necesario conocer las características principales de la peritonitis aguda postoperatoria, ya que en buena medida, el pronóstico depende de nuestras decisiones, siendo la relaparotomía precoz (4) la medida más adecuada para controlar definitivamente el foco séptico. Las características de los pacientes que actualmente se intervienen podrían favorecer la aparición de complicaciones (pacientes añosos, tumores avanzados, pluripatología, etc.), lo que nos obliga a estar alerta ante la aparición de estas.
Los objetivos de este trabajo son:

1. Describir los casos de peritonitis tras cirugía urológica que precisaron ingreso en la unidad de cuidados intensivos de nuestro hospital.

2. Describir las generalidades encontradas en la bibliografía (epidemiología, clasificación, etiopatogenia...), el manejo y tratamiento recomendado por las diferentes sociedades médicas.

3. Discutir los hallazgos de nuestra casuística a la luz de los escasos datos aportados por la bibliografía.

\section{MATERIAL Y MÉTODOS}

Se estudian retrospectivamente las cistectomías radicales realizadas entre el 1 de enero de 2002 y el 31 de enero de 2006 que presentaron en su postoperatorio peritonitis aguda. Una vez revisadas las intervenciones en este periodo, se solicitan las historias clínicas de los pacientes que requirieron reingreso en la $\mathrm{UCl}$ por peritonitis y se analizan una serie de variables clasificadas en cuatro categorías: fisiología del paciente, intervención, peritonitis y morbimortalidad.

\section{Variables relacionadas con la fisiología del paciente:}

1. Edad.

2. Sexo.

3. Analítica preoperatoria.

4. Alteración orgánica crónica previa:

- Hepática: Hepatitis, enolismo...

- Cardiovascular: Hipertensión, insuficiencia cardiaca, cardiopatía isquémica.

- Respiratoria: EPOC, SAOS, tabaquismo.

- Renal.

- Neurológica.

- Diabetes Mellitus.

- Inmunosupresión.

\section{Variables relacionadas con la intervención quirúrgi- ca:}

1. Intención quirúrgica: Curativa o paliativa.

2. Estadio tumoral (hallazgos intraoperatorios).

3. Preparación intestinal en los días previos a la intervención.

4. Tiempo quirúrgico.

5. Transfusión intraoperatoria.

6. Complicaciones intraoperatorias.

7. Tipo de intervención.

8. Anastomosis intestinal.

9. Anastomosis urétero-ileal. 
Variables relacionadas con la fisiopatología de la peritonitis:

1. Comienzo de los síntomas.

2. Datos de laboratorio.

3. Inicio de la nutrición parenteral total.

4. Localización del fallo que origina el abdomen agudo.

5. Mecanismo por el cual se desencadena el proceso.

6. Tiempo transcurrido entre el comienzo de la sintomatología y la laparotomía.

7. Tratamiento antibiótico.

8. Extensión de la peritonitis y presencia de líquido libre.

9. Necesidad de relaparotomía.

Variables relacionadas con las complicaciones postoperatorias:

1. Días de ingreso en $\mathrm{UCl}$.

2. Complicaciones infecciosas asociadas.

3. Complicaciones no infecciosas asociadas.

4. Necesidad de transfusión.

5. Causa del fallecimiento.

\section{RESULTADOS}

Entre el 1 de enero del año 2002 y el 31 de enero del 2006 se realizan un total de 80 cistectomías radicales indicadas por neoplasia vesical infiltrante en diferentes estadios. De estas 54 se realizaron con intención curativa y las 26 restantes con intención paliativa (cistectomías "de salvación"). 72 de los pacientes son varones y solo se practicó la cistectomía en 8 mujeres. La edad media de los pacientes fue de 66,68 años.

El tiempo quirúrgico medio fue $3 \mathrm{~h} 49$ minutos. En los 9 pacientes complicados fue significativamente mayor (4h 25 minutos). El tipo de derivación urinaria realizada fue Bricker en 62 pacientes, Studer en 9, Mainz II en 4 y ureterostomía cutánea en 5 .

En el plazo estudiado, 9 de las 80 cistectomías se complicaron con peritonitis $(11,25 \%)$. Todos ellos varones por encima de 50 años excepto un paciente de 44 años (edad media 62,55 años). 5 de los 9 pacientes fallecen, tres de ellos como consecuencia de la peritonitis (3,75\% de todas las cistectomías) y dos por progresión de la enfermedad.

En las Tablas I-IV se recogen los datos analizados en función de las variables comentadas anteriormente.

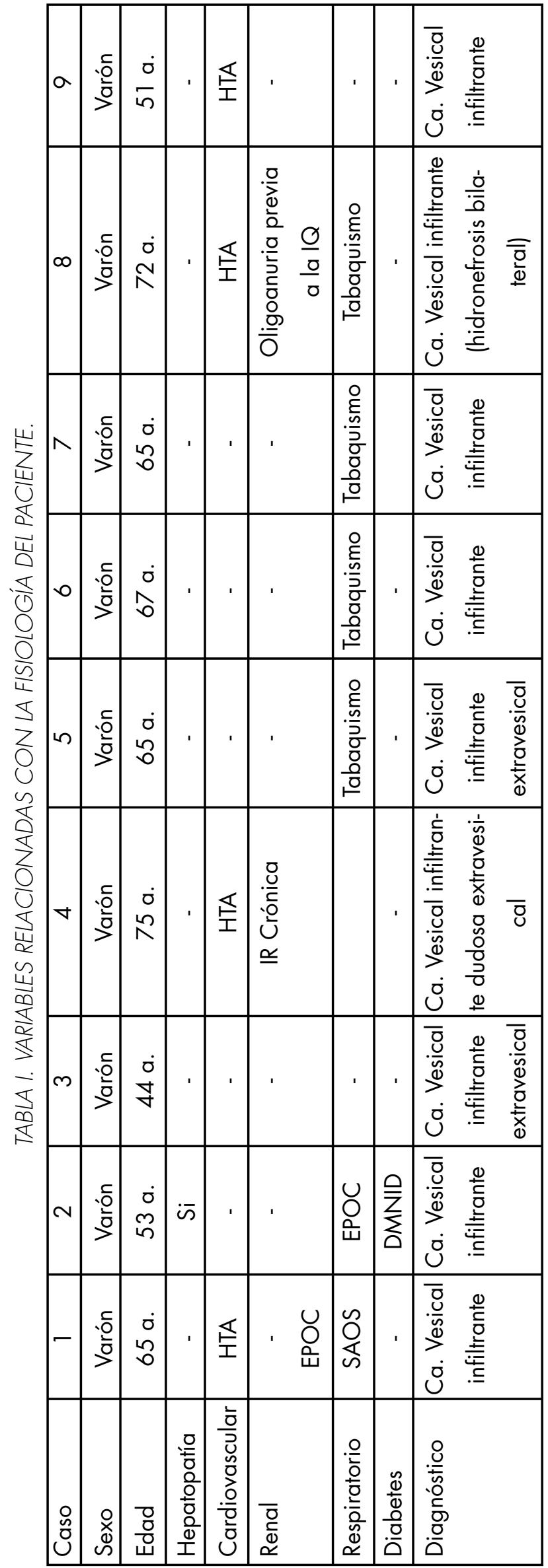




\begin{tabular}{|c|c|c|c|c|c|c|c|c|c|c|c|c|c|c|c|c|c|}
\hline$a$ & 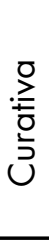 & & $\begin{array}{l}\sum_{\text {N }}^{0} \\
\text { Z } \\
\text { 임 }\end{array}$ & & is & & is & & ¿ & & 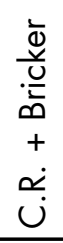 & & & $\begin{array}{l}\frac{0}{3} \\
\frac{3}{5} \\
\text { c }\end{array}$ & 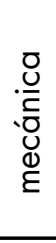 & 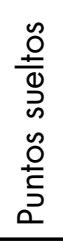 & 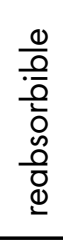 \\
\hline$\infty$ & $\frac{\bar{d}}{\frac{\delta}{0}}$ & & $\begin{array}{l}\sum_{0}^{0} \\
\text { Ż } \\
\text { \& }\end{array}$ & & is & & is & & ㅇ & & $\underset{\dot{u}}{+}$ & 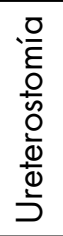 & $\begin{array}{l}0 \\
\mathbb{1} \\
.0 \\
\frac{0}{3} \\
0\end{array}$ & $\frac{0}{\frac{0}{3}}$ & 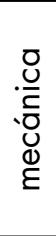 & 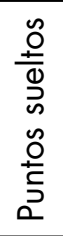 & 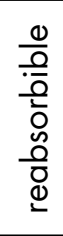 \\
\hline$\Lambda$ & 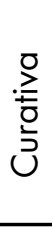 & & $\begin{array}{l}\sum_{0}^{0} \\
\text { Z } \\
\stackrel{0}{0} \\
\stackrel{N}{N}\end{array}$ & & $\bar{s}$ & & $\bar{s}$ & & ㅇ & & $\begin{array}{l}\frac{\grave{d}}{0} \\
\stackrel{D}{0} \\
+ \\
\dot{\sim} \\
\dot{u}\end{array}$ & & & $\frac{\bar{D}}{\frac{3}{5}}$ & 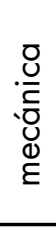 & 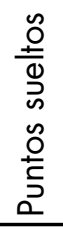 & 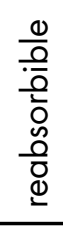 \\
\hline 0 & $\begin{array}{l}\frac{0}{2} \\
\frac{\pi}{0} \\
\stackrel{3}{J}\end{array}$ & & $\begin{array}{l}\sum_{0}^{0} \\
\text { Z } \\
\stackrel{0}{N} \\
\text { N }\end{array}$ & & $i$ & & is & & ¿ & & 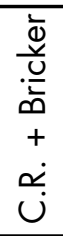 & & & $\begin{array}{l}\frac{0}{5} \\
\frac{5}{5} \\
\dot{5}\end{array}$ & 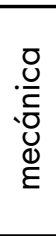 & 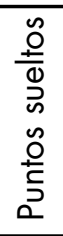 & 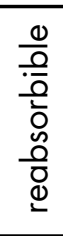 \\
\hline in & 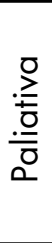 & & 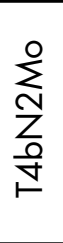 & & $i$ & & is & & $\begin{array}{l}\frac{0}{0} \\
\frac{0}{0} \\
\frac{0}{0} \\
\frac{0}{0} \\
0 \\
0\end{array}$ & 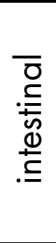 & $\begin{array}{l}+ \\
\dot{v} \\
\dot{u}\end{array}$ & 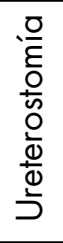 & 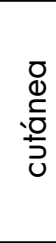 & 咅 & 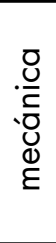 & 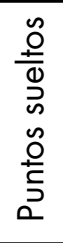 & 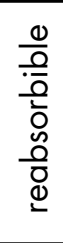 \\
\hline$\nabla$ & 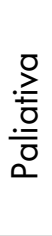 & & $\begin{array}{l}\sum_{0}^{0} \\
\text { Z } \\
\text { Oo } \\
\qquad\end{array}$ & & is & & is & & 之o & & 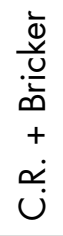 & & & 旁 & 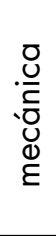 & 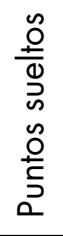 & 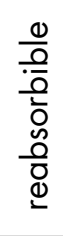 \\
\hline$m$ & 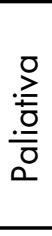 & & $\begin{array}{l}\sum_{0}^{0} \\
\text { Z } \\
\frac{0}{\square} \\
\qquad\end{array}$ & & is & & is & & ㅇ & & $\begin{array}{l}+ \\
\dot{\sim}\end{array}$ & 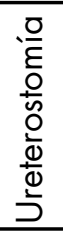 & 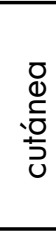 & $\frac{0}{\frac{0}{3}}$ & 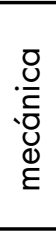 & 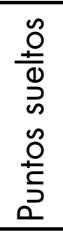 & 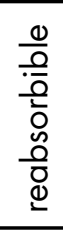 \\
\hline$\sim$ & 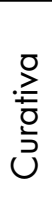 & & $\begin{array}{l}\sum_{0}^{0} \\
Z_{i} \\
\stackrel{0}{N} \\
\stackrel{1}{1}\end{array}$ & & is & & is & & ํ & & $\begin{array}{l}\frac{\grave{d}}{0} \\
\stackrel{D}{D} \\
\dot{\omega} \\
+ \\
\dot{\sim} \\
ن\end{array}$ & & & $\begin{array}{l}\frac{0}{5} \\
\frac{3}{5} \\
\dot{3}\end{array}$ & 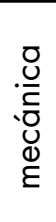 & 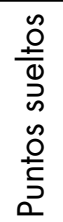 & 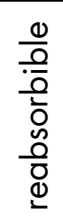 \\
\hline- & 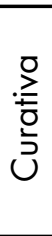 & & $\begin{array}{l}\sum_{0}^{0} \\
Z_{0} \\
\frac{0}{b}\end{array}$ & & is & & is & & ㅇ & & 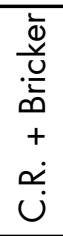 & & & 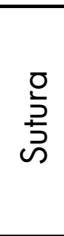 & 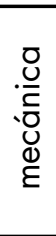 & 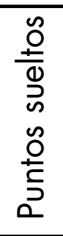 & 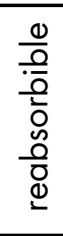 \\
\hline $\begin{array}{l}0 \\
0 \\
0 \\
0 \\
0 \\
0 \\
\text { II } \\
Z\end{array}$ & 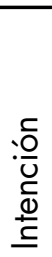 & $\begin{array}{l}\frac{0}{0} \\
\frac{3}{2} \\
\frac{2}{2} \\
\end{array}$ & $\begin{array}{l}\stackrel{\circ}{\text { N }} \\
\stackrel{0}{0} \\
\overline{\overline{0}}\end{array}$ & 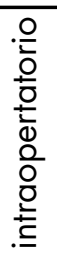 & $\begin{array}{l}\frac{0}{U} \\
\frac{0}{0} \\
\frac{0}{0} \\
\frac{0}{Q} \\
\frac{0}{2}\end{array}$ & 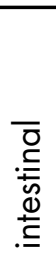 & 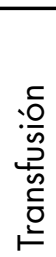 & 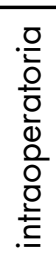 & 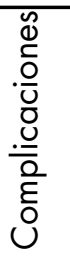 & & $\begin{array}{l}\stackrel{0}{0} \\
\stackrel{\circ}{\varrho} \\
\stackrel{\varrho}{\varrho}\end{array}$ & 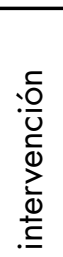 & & 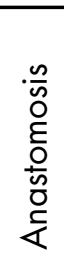 & 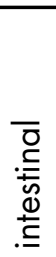 & 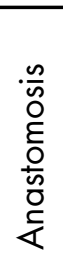 & $\begin{array}{l}\bar{\sigma} \\
\bar{\omega} \\
\bar{\omega} \\
\bar{J}\end{array}$ \\
\hline
\end{tabular}




\begin{tabular}{|c|c|c|c|c|c|c|c|c|c|c|c|}
\hline$a$ & \begin{tabular}{|l}
$\frac{0}{0}$ \\
1 \\
-1
\end{tabular} & 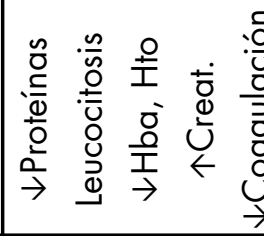 & 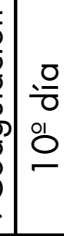 & 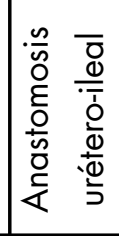 & 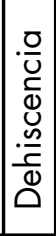 & 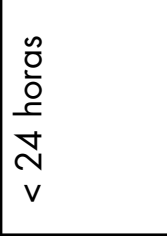 & is & 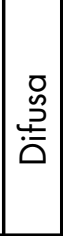 & 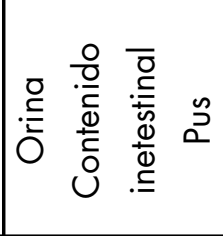 & ' & 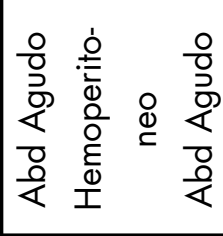 \\
\hline$\infty$ & $\begin{array}{l}\text { 응 } \\
\text { o, } \\
\text { y }\end{array}$ & 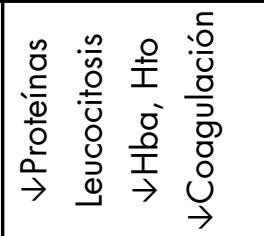 & $\begin{array}{l}\text { 응 } \\
\text { in }\end{array}$ & 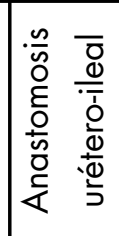 & 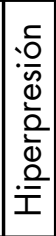 & 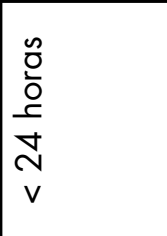 & is & 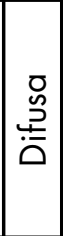 & 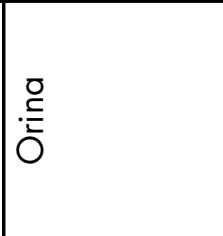 & ' & ㅇ \\
\hline$\Lambda$ & $\begin{array}{l}\frac{0}{0} \\
\frac{1}{0} \\
\stackrel{\circ}{1}\end{array}$ & 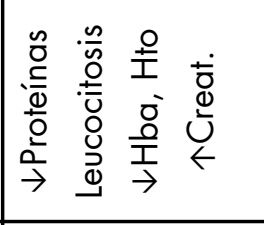 & $\begin{array}{l}\text { 응 } \\
\stackrel{\circ}{\Lambda}\end{array}$ & 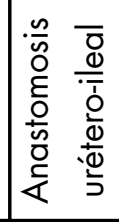 & $\begin{array}{l}\cdot \frac{0}{0} \\
\frac{C}{0} \\
0 \\
.0, \\
\frac{0}{0} \\
0 \\
0\end{array}$ & 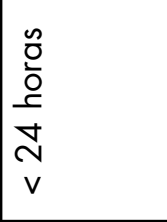 & is & 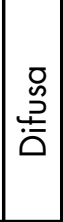 & 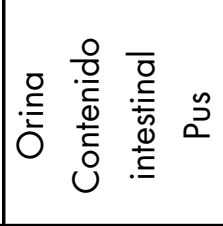 & نَ & 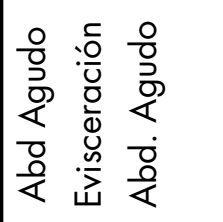 \\
\hline 0 & $\begin{array}{l}\text { 응 } \\
\text { ol } \\
\text { 이 }\end{array}$ & 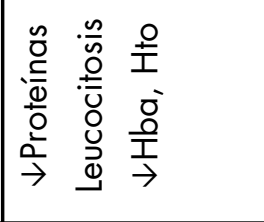 & $\begin{array}{l}\text { 응 } \\
\stackrel{\circ}{\Lambda}\end{array}$ & $\begin{array}{l}\complement \\
\delta \\
= \\
=\end{array}$ & $\left|\begin{array}{l}\frac{n}{\omega n} \\
0 \\
\frac{O}{U} \\
0 \\
Z\end{array}\right|$ & 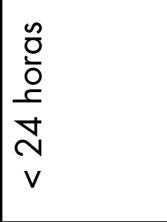 & is & 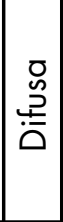 & 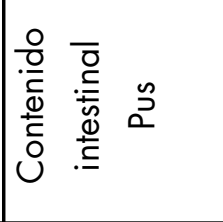 & نَ & 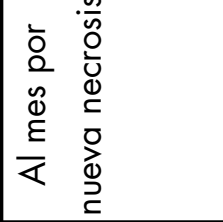 \\
\hline$n$ & $\begin{array}{l}\text { 응 } \\
\text { 임 } \\
\text { - }\end{array}$ & 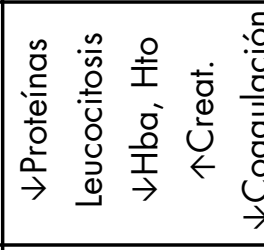 & 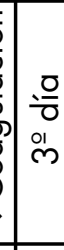 & 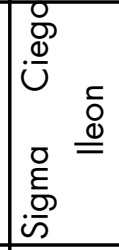 & 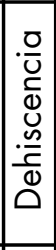 & 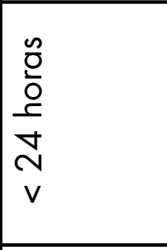 & is & 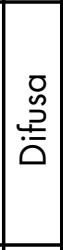 & 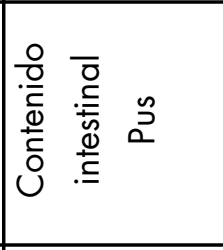 & ' & Z \\
\hline$\nabla$ & $\begin{array}{l}\frac{0}{0} \\
\frac{0}{0} \\
\infty \\
\infty\end{array}$ & 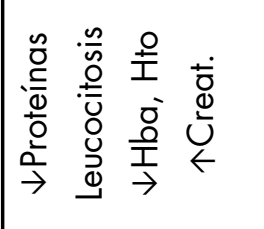 & $\begin{array}{l}i \frac{0}{0} \\
\text { on } \\
\text { in }\end{array}$ & 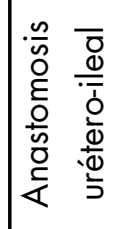 & 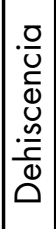 & 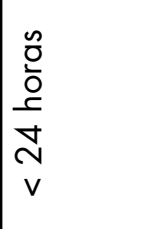 & is & 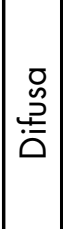 & 宑 & ' & 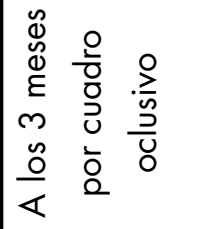 \\
\hline$m$ & $\begin{array}{l}\text { 응 } \\
\text { 이 } \\
\text { लn }\end{array}$ & 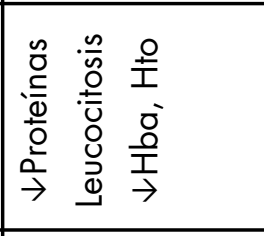 & & 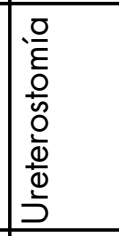 & 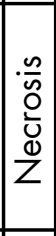 & 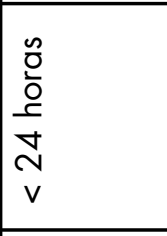 & $i \bar{s}$ & 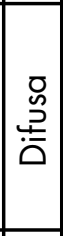 & 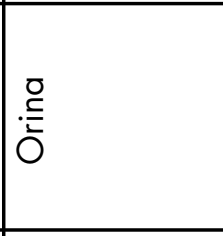 & 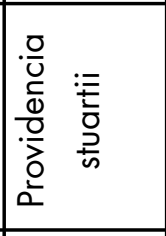 & ㅇ \\
\hline$\sim$ & 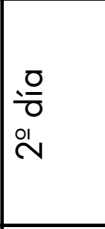 & 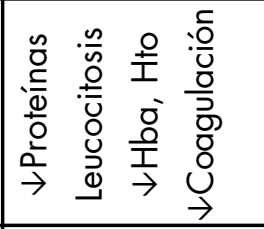 & $\begin{array}{l}\text { 음 } \\
\text { 이 }\end{array}$ & 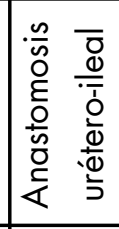 & \begin{tabular}{|c|}
$\cdot \frac{0}{0}$ \\
$\frac{c}{0}$ \\
0 \\
.0, \\
$\frac{0}{0}$ \\
0 \\
0 \\
\end{tabular} & 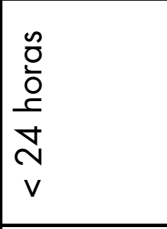 & $i \bar{s}$ & 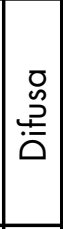 & 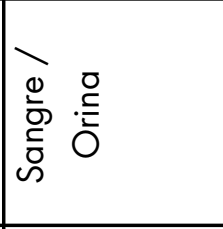 & ' & 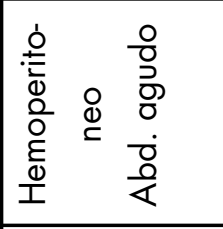 \\
\hline- & 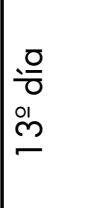 & 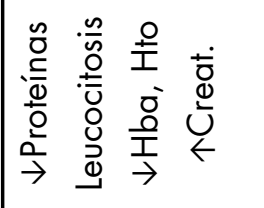 & $\begin{array}{l}\text { 음 } \\
\text { 이 }\end{array}$ & 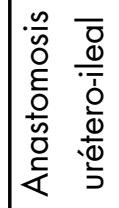 & 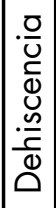 & $\begin{array}{l}\frac{\mathscr{y}}{0} \\
\frac{i}{0} \\
\gamma\end{array}$ & is & 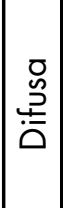 & : & 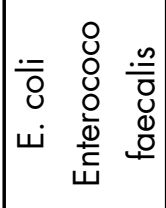 & ㅇ \\
\hline $\begin{array}{l}0 \\
0 \\
0 \\
0 \\
0 \\
0 \\
\text { ol } \\
Z\end{array}$ & 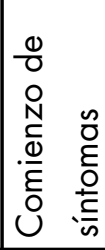 & 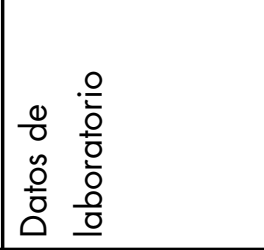 & 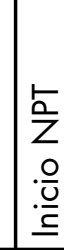 & 离 & 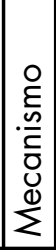 & 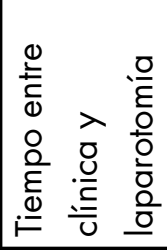 & $\begin{array}{l}.0 \\
\frac{0}{2} \\
\frac{d}{2} \\
2 \\
\frac{2}{\alpha}\end{array}$ & \begin{tabular}{|c|}
$: \frac{c}{0}$ \\
0 \\
$\frac{0}{0}$ \\
$\frac{0}{x}$ \\
$w$
\end{tabular} & 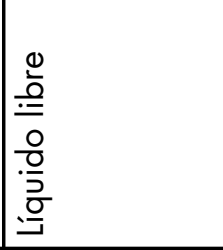 & 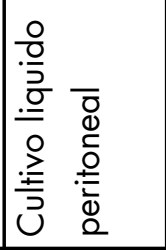 & 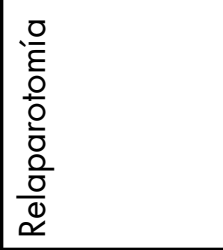 \\
\hline
\end{tabular}




\begin{tabular}{|c|c|c|c|c|c|c|c|c|c|c|c|c|c|c|c|c|c|c|}
\hline$a$ & $\stackrel{a}{-}$ & $\begin{array}{l}\frac{0}{0} \\
\frac{0}{0} \\
\frac{1}{1}\end{array}$ & $\begin{array}{l}\frac{0}{0} \\
\frac{0}{3} \\
\frac{3}{3} \\
\frac{\partial}{\sigma}\end{array}$ & $\begin{array}{l}\stackrel{0}{O} \\
\text { O } \\
\text { Eे } \\
\text { d } \\
\text { Z }\end{array}$ & 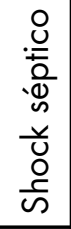 & 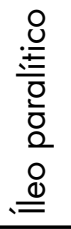 & 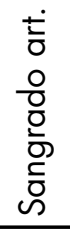 & 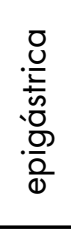 & $\begin{array}{l}\text { u } \\
\frac{c}{v} \\
\end{array}$ & 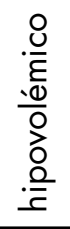 & $\frac{\frac{0}{2}}{\frac{2}{50}}$ & 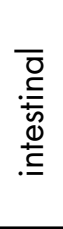 & & is & $\begin{array}{l}0 \\
\sim \\
N\end{array}$ & 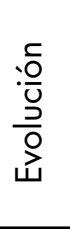 & $\begin{array}{l}0 \\
00 \\
0 \\
0 \\
.0 \\
0 \\
0 \\
.0\end{array}$ & 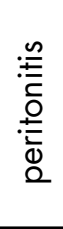 \\
\hline$\infty$ & $\Lambda$ & ' & & & & 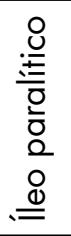 & & & & & & & & $i \bar{s}$ & ㅇ & ' & & \\
\hline$\Lambda$ & $\stackrel{m}{-}$ & 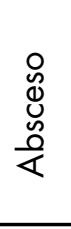 & 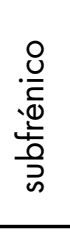 & $\begin{array}{l}\frac{0}{0} \\
\frac{0}{0} \\
\frac{0}{1}\end{array}$ & $\begin{array}{l}\frac{0}{0} \\
\frac{0}{2} \\
\frac{2}{3} \\
\bar{\sigma}\end{array}$ & 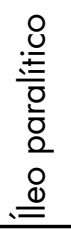 & $\begin{array}{l}\stackrel{0}{E} \\
\stackrel{0}{0} \\
\stackrel{2}{0} \\
\square\end{array}$ & $\begin{array}{l}\overline{0} \\
\overline{\frac{0}{0}} \\
\end{array}$ & $\begin{array}{l}\frac{0}{0} \\
\frac{0}{U} \\
\frac{d}{0} \\
\frac{0}{d}\end{array}$ & & & & & $i \bar{s}$ & ㅇ & & & \\
\hline 0 & 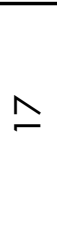 & 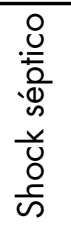 & & & & 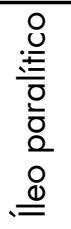 & & & & & & & & $\bar{n}$ & $\begin{array}{l}\text { o } \\
\ddot{n}\end{array}$ & 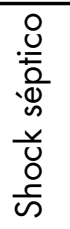 & & \\
\hline in & $\stackrel{\infty}{-}$ & 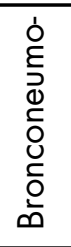 & 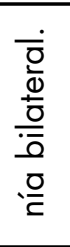 & 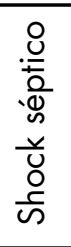 & & $\frac{0}{\frac{0}{2}}$ & $\begin{array}{l}\frac{0}{\overline{0}} \\
\frac{0}{0} \\
\frac{0}{0} \\
3 \\
0 \\
0 \\
0\end{array}$ & 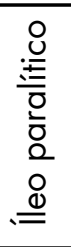 & & & & & & is & $\begin{array}{l}\text { ర் } \\
\infty \\
-\end{array}$ & 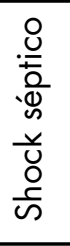 & & \\
\hline$\nabla$ & $\stackrel{\infty}{m}$ & ' & & & & 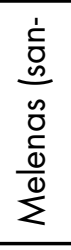 & $\begin{array}{l}\bar{n} \\
\bar{u} \\
J \\
0 \\
0 \\
0 \\
0 \\
0\end{array}$ & $\frac{0}{\frac{0}{5}}$ & $\begin{array}{l}\frac{0}{\overline{0}} \\
0 \\
0 \\
0 \\
0 \\
0 \\
0 \\
\\
\end{array}$ & 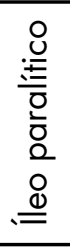 & 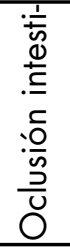 & 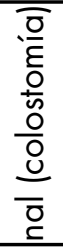 & & is & $\begin{array}{l}\dot{\varepsilon} \\
\sim \\
\dot{\nabla}\end{array}$ & $\begin{array}{l}: \frac{0}{\mathscr{N}} \\
\stackrel{0}{0} \\
\stackrel{0}{0} \\
\stackrel{0}{2}\end{array}$ & 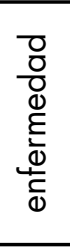 & \\
\hline m & ㄱ & 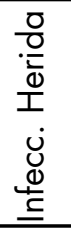 & 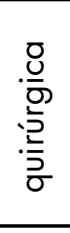 & & & 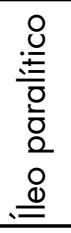 & & & & & & & & is & $\underset{-}{\dot{0}}$ & 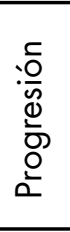 & 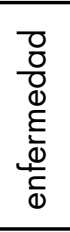 & \\
\hline$\sim$ & $\stackrel{m}{m}$ & $\begin{array}{l}\frac{0}{0} \\
\frac{0}{0} \\
\frac{1}{I}\end{array}$ & $\begin{array}{l}\frac{0}{0} \\
\frac{0}{2} \\
\frac{\underline{n}}{\partial}\end{array}$ & 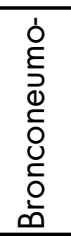 & $\frac{\sigma}{c}$ & 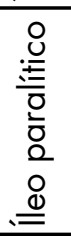 & $\begin{array}{l}\frac{y}{U} \\
\frac{o}{\omega}\end{array}$ & 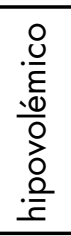 & $\begin{array}{l}\frac{0}{5} \\
\frac{2}{4}\end{array}$ & $\begin{array}{l}\frac{0}{7} \\
\frac{0}{0} \\
0 \\
0 \\
0 \\
0 \\
0 \\
0 \\
0\end{array}$ & $\frac{0}{\frac{0}{5}}$ & 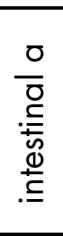 & $\overline{\frac{\omega}{\alpha}}$ & $\overline{i s}$ & ㅇ & . & & \\
\hline- & m) & $\begin{array}{l}\frac{\infty}{ \pm} \\
\frac{0}{0} \\
\frac{0}{4}\end{array}$ & 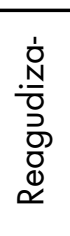 & 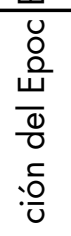 & & 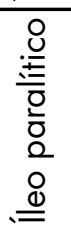 & $\begin{array}{l}\overline{\bar{O}} \\
\bar{D} \\
\stackrel{\alpha}{0} \\
\dot{-}\end{array}$ & $\begin{array}{l}\frac{0}{0} \\
\frac{8}{8}\end{array}$ & & & & & & is & ㅇ & . & & \\
\hline $\begin{array}{l}0 \\
0 \\
0 \\
0 \\
0 \\
0 \\
\text { OI }\end{array}$ & 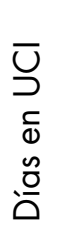 & $\frac{\frac{\dot{U}}{\alpha}}{\stackrel{\varrho}{\underline{O}}}$ & 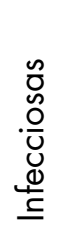 & & & $\frac{\frac{\dot{u}}{\alpha}}{\stackrel{\varrho}{\varepsilon}}$ & 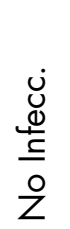 & & & & & & & 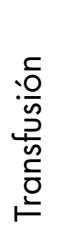 & 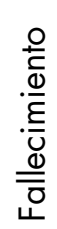 & $\begin{array}{l}\overline{0} \\
0 \\
0 \\
\tilde{O} \\
\tilde{U}\end{array}$ & 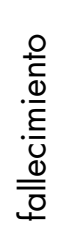 & \\
\hline
\end{tabular}


En cuanto a la patología previa no presentaban un estado general especialmente deteriorado: 4 de los 9 son fumadores importantes; 4 hipertensos en tratamiento médico; un hepatópata y un diabético. 4 de los pacientes fueron diagnosticados de afectación extravesical.

Previa a la intervención, todos los pacientes ingresan con tiempo suficiente para realizar la preparación intestinal y administrar la profilaxis antibiótica adecuada. La intención quirúrgica fue curativa en 5 de los pacientes y en 4 paliativa. Todos los pacientes estudiados precisaron transfusión intraoperatoria y tan sólo uno de ellos presentó complicaciones intraoperatorias (perforación intestinal). En cuanto a la técnica practicada, de los 9, 4 fueron cistectomías radicales con derivación urinaria tipo Bricker, a 3 se les realizó ureterostomía cutánea y a 2, neovejiga ortotópica tipo Studer. La anastomosis intestinal se realiza en todos los casos con sutura mecánica y la ureteral mediante puntos sueltos reabsorbibles. En cuatro de los pacientes se inició la nutrición parenteral total dentro de la primera semana.

El comienzo de los síntomas ocurrió en 4 ocasiones antes de la primera semana tras la intervención, y la laparotomía exploradora se realizó en las primeras 24 horas tras la aparición de la clínica en todos excepto en un paciente. Las alteraciones analíticas comunes fueron un descenso evidente del nivel sérico de proteínas, leucocitosis y anemia, que en algunas ocasiones preciso transfusión.

Los hallazgos en la laparotomía evidenciaron que el origen de la peritonitis fue el fracaso de la anastomosis intestinal en 2 casos, 1 en la ureterostomía cutánea y en 6 pacientes en la anastomosis urétero-ileal. El mecanismo que originó el fallo fue en 6 ocasiones la dehiscencia de la anastomosis, en 2 casos por necrosis y en uno de ellos la hiperpresión dentro de la vía urinaria. La extensión de la inflamación en ninguno de los casos fue focal, existiendo peritonitis difusa en la totalidad de los pacientes. El exudado intraabdominal fue variable, apareciendo asociado el exudado purulento con el contenido intestinal en 4 de los casos y orina en el resto. Se enviaron muestras a microbiología de 4 pacientes, obteniendo resultado positivo para E. coli en 3 pacientes (además de Enterococo faecalis en uno de ellos) y Providencia stuartii en el otro cultivo. La necesidad de relaparotomizar al paciente sucedió en 3 pacientes dentro del primer mes (hemoperitoneo, síndrome compartimental intraabdominal, ...) y en dos a partir de este plazo (cuadro oclusivo por adherencias y por necrosis, respectivamente).
Las complicaciones asociadas las clasificamos en dos grupos en función del origen, así, dentro de las infecciosas, en 4 pacientes la focalización fue respiratoria, en otros 4 la infección afectó a la herida quirúrgica y 1 caso de absceso subfrénico (drenado mediante punción ecodirigida). Dentro de las complicaciones no infecciosas se incluyen: íleo paralítico (100\% de los casos); insuficiencia renal y anuria $(4$ casos); coagulopatía (3 pacientes); shock hipovolémico (2 casos); derrame pleural y atelectasia (1 caso) y oclusión intestinal con necesidad de colostomía en un paciente.

Cinco de los 9 pacientes fallecieron: en dos pacientes la causa fue la progresión de la neoplasia (al año y a los 4,5 meses respectivamente) y tres fallecieron a los 18,25 y 35 días propiamente por shock séptico secundario a la peritonitis aguda $(3,75 \%$ del total de cistectomías).

\section{DISCUSIÓN}

Como se mencionó anteriormente, en la literatura médica no es fácil encontrar artículos que hagan referencia de forma explícita al tema que nos ocupa, por lo que no ha sido posible comparar nuestros resultados con los de otras series. Además, el tamaño de nuestra muestra no se considera lo suficientemente amplio como para poder extrapolar los resultados.

No obstante la gravedad de esta complicación y la mortalidad que alcanza justifican el hecho que se intenten extraer conclusiones para poder anticiparse a la aparición de ésta y en caso de que esto ocurra podamos abordarla con la eficacia que merece. De hecho, la mortalidad alcanza el $50 \%$ cuando se originan en el postoperatorio de una cirugía abdominal. Un $50 \%$ de estos pacientes desarrollan un fracaso multiorgánico. El transcurso de este proceso vendrá influenciado por la reserva fisiológica del paciente, la severidad y tipo de enfermedad, el éxito de la intervención y manejo posterior y las posibles complicaciones $(1,3,11)$.

Afortunadamente en nuestra especialidad, la incidencia no es tan elevada como lo es en otras, las intervenciones practicadas sobre el aparato digestivo son las que presentan con más frecuencia esta complicación, estimada según autores en torno al $20 \%$. Por el contrario, las practicadas sobre la vía urinaria, analizadas globalmente, raramente se complican con un cuadro peritonítico. De hecho, en nuestro Servicio tan sólo hemos presenciado esta complicación en la cistectomía radical, no existiendo en ningún otro tipo de intervención. Sin embargo en la bibliografía si hemos podido encontrar escritos que citen la apari- 
ción de un abdomen agudo en otras intervenciones diferentes de la cistectomía, como por ejemplo:

- Peritonitis biliar tras nefrolitotomía percutánea o tras la colocación de nefrostomía $(21,23)$ : El decúbito supino ligeramente lateralizado mediante una bolsa de suero en la fosa lumbar del lado afecto ha disminuido los casos de iatrogénica en nuestro servicio.

- Peritonitis post biopsia transrectal de próstata (22): Peritonitis fecaloidea confirmada mediante la laparotomía secundaria a fístula recto-peritoneal.

- Sobreinfección de esfínter artificial tipo AMS-800 que originó un abdomen agudo (19).

- Rotura espontánea de neovejiga ortotópica $(24$, 25). Se describe el caso de un paciente al que la sobredistensión vesical secundaria a la retención aguda de orina por un tapón de moco en la desembocadura a uretra provoca el estallido vesical.

- Lesión iatrogénica de vísceras abdominales durante la cirugía urológica. Según la literatura médica, dentro de este apartado lo que ocurre con más frecuencia es la perforación rectal durante la prostatectomía radical (20), teniendo en cuenta la íntima relación anatómica entre la pared anterior del recto y la cara posterior de la próstata, vejiga y vesículas seminales. Concretamente en la prostatectomía radical laparoscópica el porcentaje de lesiones rectales oscila entre el $0,3 \%$ y el $9 \%$.

Se han hecho múltiples estudios intentando identificar factores de riesgo asociados a la mortalidad en peritonitis y sepsis abdominal que ayuden a la toma de decisiones terapéuticas, incluyendo índices pronósticos o sistemas de valoración de la gravedad. Así, Bosscha y col. determinan entre varios sistemas, que Acute Physiology and Chronic Health Evaluation (APACHE II) y Mannhein Peritonitis Index (MPI) (5) son los que mejor predicen la mortalidad relacionada con la peritonitis. Los estudios que valoran los factores pronósticos analizan una serie de variables que determinan fundamentalmente el estado de salud previo del paciente, la severidad y tipo de enfermedad y las complicaciones intra y postoperatorias (12).

Wacha y cols. estudiaron los factores de riesgo de mortalidad y complicaciones postoperatorias, siendo significativos de forma independiente la edad, la presencia de enfermedad concomitante (hepatopatía, enfermedad renal, neoplasia), el APACHE II y el éxito de la operación (9). Wahlm y col consideran factores pronósticos de peritonitis la extensión y la localización, la formación de abscesos, el número de órganos alterados y el descenso en sangre de proteínas totales, plaquetas y leucocitos. Barhlenw y cols. Estudian factores pronósticos de peritonitis difusa, siendo significativos la edad, la presencia de hepatopatía, el aumento de la creatinina sérica y lo imposibilidad de erradicación del foco infeccioso en la primera intervención quirúrgica (16). Pacelli y cols. (3) encuentran asociados significativamente a la mortalidad por infección intraabdominal el descenso de la albúmina y colesterol en suero, la alteración orgánica preoperatoria, el APACHE II y el MPI. Por otro lado, Koperna y col. (6) consideran importante el APACHE II mayor de 15, el número de órganos afectados, la Diabetes Mellitus, la trombocitopenia inicial, la peritonitis difusa, el tratamiento inadecuado y la necesidad de relaparotomía.

En la literatura médica no es fácil encontrar un estudio que se centre selectivamente en las causas y factores que influyen en el desarrollo de peritonitis tras un procedimiento urológico. Lógicamente, los factores citados anteriormente en las peritonitis $\sin$ especificar la intervención realizada son aplicables a las peritonitis tras intervencionismo urológico.

Es importante recordar que teniendo en cuenta la escasa cantidad de pacientes recogidos en nuestro estudio no se pueden extraer conclusiones estadísticas extrapolables.

\section{CONCLUSIONES}

Sería lógico pensar que la aparición de esta entidad aumentara con la edad del paciente y con la patología asociada. Esto nos lleva a pensar que debido al tipo de pacientes que se están interviniendo actualmente (pacientes añosos, con pluripatología, con tumores avanzados,...) la incidencia de la peritonitis postcistectomía aumentará

Una incidencia del 11,25\% de las cistectomías realizadas durante cinco años no es ni mucho menos despreciable, teniendo en cuenta la gravedad del problema. Si contabilizamos el número total de intervenciones urológicas, recordando que la mayoría son extraperitoneales (prostatectomías, adenomectomías, pieloplastias, biopsias prostáticas, nefrostomías, nefrolitectomías percutáneas, etc.), la incidencia es mínima.. Es cierto que en estos postoperatorios existe excepcionalmente mínima fuga urinaria que podría provocar clínica peritoneal, pero no ha sido necesario realizar una laparotomía para solucionarla.

Es importante reconocer precozmente un abdomen agudo, identificar la causa y actuar en consecuencia, ya que, generalmente, la progresión 
de la enfermedad conduce hacia la sepsis con el consecuente fracaso multiorgánico. Del mismo modo es importante reconocer con antelación al procedimiento quirúrgico los factores de riesgo que pueden influir para desarrollar la peritonitis. Aunque no podemos con certeza anticiparnos a esta complicación, podemos analizar las características del paciente y vigilar su evolución. Entre los factores, debemos tener en cuenta, según nuestra experiencia: la edad del paciente, el estado orgánico previo a la intervención (considerando de forma especial la hepatopatía, las alteraciones renales y la inmunosupresión), la extensión de la enfermedad neoplásica, otras complicaciones postoperatorias, el tratamiento adecuado, el nivel sérico de proteínas y creatinina y la actividad de protrombina.

\section{BIBLIOGRAFÍA y LECTURAS RECOMENDADAS ( ${ }^{*}$ lectura de interés $y^{* *}$ lectura fundamental)}

**1. WITTMANN, H.; SCHEIN, M.; CONDON, R.: "Management of secondary Peritonitis". Ann. Surg., 224: 10, 1996.

*2. PICAZO, J.; KARAM, G.; GARCÍA RODRÍGUEZ, J. y cols.: "Tratamiento antimicrobiano empírico de las peritonitis". Inf. Dis. Clin. Pract., 31-33, 2004.

**3. PACELLI, F.; DOGLIETTO, G.; ALFIERI, S. y cols.: "Prognosis in intra-abdominal infections: Multivariate analysis on 604 patiens". Arx. Surg., 131: 641, 1996.

**4. LAMME, B.; BOERMEESTER, M.A.; REITSMA, J.B. y cols.: "Meta-analysis of relaparotomy for secondary peritonitis". Br. J. Surg., 89: 1516, 2002.

*5. FARIÑAS-ALVAREZ, C.; FARIÑAS, M.C.; LLORCA, J. y cols.: "Analysis of risk factors for nosocomial sepsis in surgical patiens". Br. J. Surg., 87: 1076, 2000.

6. KOPERNA, T.; SCHULZ, F.: "Prognosis and treatament of peritonitis: Do we need new scoring systems?". Arch. Surg., 131: 180, 1996.

7. NATHENS, A.; ROTSEIN, O.; MARSAHALL, J.: "Tertiary peritonitis: Clinical Features of a complex nosocomial infection". World J. Surg., 22: 158, 1998.

**8. MAZUSKI, J.; SAWYER, R.; NATHENS, A.S. y cols.: "The surgical infection society guidelines on antimicrobial therapy for intra-abdominal infections: an executive summary". Surg. Inf., 3: 161, 2002.

9. SHINKAWA, H.; YASUHARA, H.; NAKA, S. y cols.: "Factors affecting the early mortality of patiens with nontraumatic colorectal perforation". Surg. Today, 33: 33, 2002.
10. BUIJK, S.; BRUINIG, H.: "Future directions in the management of tertiary peritonitis". Intensive Care Med., 28: 1024, 2002.

*11. MARSHALL, J.; INNES, M.: "Intensive care unit management of intra-abdominal infection". Crit. Care Med., 31: 2228, 2003.

*12. MULIER, S.; PENNINCKX, F.; VERWAEST, C. y cols.: "Factors affecting mortality in generalized postoperative peritonitis: Multivariate analysis in 96 patients". World J. Surg., 27: 379, 2003.

**13. SOLOMKIM, J.; MAZUSKI, J.; BARON, E. y cols.: "Guidelines for selection of anti-infective agents for complicated intra-abdominal infections". Clin. Infec. Diseases, 37: 997, 2003.

14. EACHEMPATI, S.; LYDO, L.; BARIE, P.: "Gender-Based diffences in outcome in patiens with sepsis”. Arch. Surg., 134: 1342, 1999.

15. CRABTREE, T.; PELLETIER, S.; GLEASON, T. y cols.: "Gender-Dependent differences in outcome after tye treatment infection in hospitalized patiens". JAMA, 282: 2143, 1999.

16. WICHMANN, M.; INTHORN, D.; ANDRESS, H. y cols.: "Incidence and mortality of severe sepsis in surgical intensive care patiens: The influence of patient gender on disease process and outcome". Intensive Care Med., 26: 167, 2000.

17. BOCHUD, P.: "Pathogenesis of sepsis: New concepts and implications for future treatment". BMJ, 326: 262, 2003.

18. HOTCHKISS, R.; KARL, I.: "The pathophysiology and treatment of sepsis". N. Engl. J. Med., 348: 138, 2003.

19. STEFANI, S.; LIGUORI, G.; CIAMPALINI, S. y cols.: "AMS 800 Articicial sphincter: An unusual case of circumscribed peritonitis due to prosthethic reservoir infection". Arch. Esp. Urol., 52: 412, 1999.

20. GUILLONNEAU, B.; GUPTA, R.; FETTOUH, H. y cols.: "Laparoscopic manegement of rectal injury during laparoscopic radical prostatectomy". J. Urol., 169: 1694, 2003.

21. FISHER, M.; BIANCO, F.; CARLIN, A. y cols.: "Biliary peritonitis complicating percutaneous nephrolithotomy requiring laparoscopic cholecystectomy". J. Urol., 171: 791, 2004.

22. DA SILVA, E.; PEREIRO, B.; GARIMALDI, B. y cols.: "Peritonitis post biopsia transrectal de próstata". Arch. Esp. Urol., 52: 167, 1999.

23. MARÍN, E.; LUJÁN, M.; PÁEZ, A. y cols.: "Puncture of the gall bladder: an unusual cause of peritonitis complicating percutaneous nephrostomy". Br. J. Urol., 77: 464, 1996.

24. THOMPSON, S.; KURSH, E.: "Delayed spontaneous rupture of an ileocolonic neobladder". J. Urol., 148: 1890, 1992.

25. BURGOS, R.; SÁNCHEZ-BERNAL, C.; MARTÍNEZ, M.: "Rotura espontánea de vejiga: un caso". Actas Urol. Esp., 3: 107, 1979. 